\title{
Case Report: Paroxysmal nocturnal hemoglobinuria in a
}

\section{woman heterozygous for G6PD A-[version 1; peer review: 2}

\section{approved with reservations]}

\author{
Nieves Perdigones¹, Mariela Morales¹, Philip Mason¹, Monica Bessler ${ }^{1,2}$ \\ ${ }^{1}$ Division of Hematology, Department of Pediatrics, Abramson Research Center, The Children's Hospital of Philadelphia, \\ Philadelphia, PA, 19104, USA \\ 2Division of Hematology, University of Pennsylvania School of Medicine, Philadelphia, PA, 19104-4318, USA
}

V1 First published: 13 Aug 2014, 3:194
https://doi.org/10.12688/f1000research.4980.1

Latest published: 21 Oct 2014, 3:194

https://doi.org/10.12688/f1000research.4980.2

\section{Open Peer Review}

\begin{tabular}{lccc} 
Approval Status & $?$ \\
& 1 & 2 & 3 \\
\hline version 2 & & & \\
(revision) & & & \\
21 Oct 2014 & & & \\
version 1 & $?$ & view \\
13 Aug 2014 & view & view & \\
\end{tabular}

1. Rosario Notaro, Istituto Toscano Tumori, Florence, Italy

\section{Anastasios Karadimitris, Imperial College Healthcare NHS Trust and Imperial College, London, UK
3. José M. Bautista, Complutense University of Madrid, Madrid, Spain

Any reports and responses or comments on the article can be found at the end of the article. 
Corresponding author: Philip Mason (masonp@email.chop.edu)

Competing interests: No competing interests were disclosed.

Grant information: The work has been supported by the Buck Family Endowed Chair in Hematology, and by NCI NIH grants 2R01CA106995 to PJ Mason, and 2R01 CA105312 to M Bessler.

The funders had no role in study design, data collection and analysis, decision to publish, or preparation of the manuscript.

Copyright: @ 2014 Perdigones N et al. This is an open access article distributed under the terms of the Creative Commons Attribution License, which permits unrestricted use, distribution, and reproduction in any medium, provided the original work is properly cited. Data associated with the article are available under the terms of the Creative Commons Zero "No rights reserved" data waiver (CC0 1.0 Public domain dedication).

How to cite this article: Perdigones N, Morales M, Mason P and Bessler M. Case Report: Paroxysmal nocturnal hemoglobinuria in a woman heterozygous for G6PD A- [version 1; peer review: 2 approved with reservations] F1000Research 2014, 3:194 https://doi.org/10.12688/f1000research.4980.1

First published: 13 Aug 2014, 3:194 https://doi.org/10.12688/f1000research.4980.1 


\section{Introduction}

In paroxysmal nocturnal hemoglobinuria ( $\mathrm{PNH})$ one or more clones of blood cells develops from stem cells that have an acquired mutation in the $\mathrm{X}$-linked PIGA gene ${ }^{1}$. The PIGA gene encodes phosphatidylinositol glycan complementation class A, an enzyme that catalyses an early and essential step in glycosylphosphatidylinositol (GPI) anchor synthesis. Thus cells are deficient in all GPI anchored proteins, including CD55 and CD59 which regulate complement activation. PNH usually develops in patients with aplastic anemia (AA) and it is thought that PNH cells have a growth or survival advantage over the AA cells although the mechanism is not known ${ }^{2}$. PNH cells can be completely deficient in GPI anchored proteins (Type III) or partially deficient due to residual activity of the PIGA protein (Type II), while PNH Type I cells express GPI-linked proteins normally.

Clinically, PNH is characterized by bone marrow failure, thrombosis and intravascular hemolysis. Recently the use of a complement inhibitor, eculizumab has greatly improved the quality of life of PNH patients as it causes a dramatic reduction in the hemolysis and thrombotic episodes, improvement in anemia, with a stabilization of the hemoglobin levels and reduced transfusion requirements ${ }^{3}$. Eculizumab leads to an increase in the number of circulating red blood cells that otherwise are subject to complement-mediated hemolysis ${ }^{4}$.

Glucose-6-Phosphate Dehydrogenase (G6PD) deficiency is the most common red blood cell enzymopathy and is estimated to affect around 400 million people worldwide ${ }^{5}$. It is caused by mutations in the X-linked G6PD gene which usually lead to an unstable enzyme. G6PD is needed to maintain NADPH and consequently reduced glutathione levels in red blood cells. G6PD-deficient people, mainly males, can be asymptomatic but are subject to episodes of hemolysis when the red blood cells are subjected to oxidative stress caused by infections, certain drugs or in the case of favism, after eating fava beans ${ }^{6}$. Several polymorphic variants have been described with specific geographical distributions ${ }^{7}$. In the African population the most common deficient variant is the G6PD A- variant. Compared with normal G6PD, which is called G6PD B, G6PD A- has two amino acid substitutions Val68Met and Asn $126 \mathrm{Asp}^{8}$. These are caused by mutations c.202 G->A and c.376A->G respectively. G6PD A- has a frequency of about $10 \%$ in Africans and African Americans. G6PD A differs from G6PD B only by the Asn126Asp change and is electrophoretically distinct but with no significant difference in activity. Though milder than other variants such as G6PD Mediterranean found in Italy, Greece and India, G6PD A- is associated with drug induced hemolysis and patients are advised against taking any substances from a list of those known to cause hemolysis. G6PD deficiency usually only affects hemizygous males and homozygous females but heterozygous females can be affected when, for example, biased $\mathrm{X}$-inactivation has led to a predominance of red blood cells expressing the mutant protein'. Here we present a case of an African American woman who was heterozygous for G6PD deficiency and developed PNH, presenting an opportunity to observe the interaction of these two conditions.

\section{Materials and methods}

Peripheral blood from patient CHOP277.01 was obtained after obtaining written informed consent according to the declaration of
Helsinki. The Internal Review Board of the Hospital of the University of Pennsylvania approved this study. DNA and RNA were extracted by using QIAamp DNA and RNA Blood mini Kits, respectively, according to manufacturers' instructions. Blood samples for fluorescent cytometry and electrophoretic analyses were obtained from EDTA tubes and experiments were performed within 2 hours of blood withdrawal.

PCR primers to detect mutations confirming the G6PD A- genotype were designed with Primer3 v4.0. (primers for c.202 G->A mutation: forward 5'- agaagaagatctaccccaccatct-3' and reverse 5'ctggtacagagggcagaaccag-3'; primers for c.376A->G: forward 5'-catctgtctgtgtgtctgtctgtc-3' and reverse 5'- ctcatagagtggtgggaggac-3'). Sanger sequencing was done by the Nucleic Acids core facility at CHOP.

The HUMARA assay was performed as previously described ${ }^{10}$. Briefly, HhaI digested and non digested DNA was subjected to PCR amplification of the first exon of the HUMARA locus (containing a CAG repeat) using fluorochrome-coupled primers. Amplification products were then migrated on an ABI PRISM 3100 Automatic Genetic Analyzer (Applied Biosystems). Allele calling and the area under the curve (AUC) were determined using GeneMapper v.4.0 software (Applied Biosystems). The AUC was used to calculate the skewing from $\mathrm{X}$ chromosome inactivation (XCI) The XCI ratio of the digested fraction was corrected with that of the undigested fraction to allow for preferential amplification of the smallest allele (i.e., the allele containing less CAG repeats). Skewing is present when the percentage of the predominant allele exceeds $74 \%$. A percentage of predominant allele between $90 \%$ and $100 \%$ is considered extreme skewing.

Measurements of oxidative stress ROS assay was performed as previously described ${ }^{11}$. Briefly, red blood cells were incubated with 20-70-dichlorofluorescein diacetate (DCF; Sigma) dissolved in methanol. After incubation at $37^{\circ} \mathrm{C}$ for 15 minutes in a humidified atmosphere of 5\% $\mathrm{CO} 2$ in air, the cells were washed, resuspended in PBS and analyzed by flow cytometry (FACSCalibur; Becton-Dickinson, Immunofluorometry Systems, Mountain View, CA, USA). The mean fluorescence channel (MFC) was calculated by FACSDiva software. The identity of the red cell population was verified by staining with an antibody anti to glycophorin-A. To determine the presence of GPI proteins, cells were labeled with a phycoerythrin-conjugated anti-CD55 antibody. For our experiment, cells from a non PNH- non G6PD individual served as control. The MFC of cells stained with DCF, is proportional to generation of ROS.

The electrophoretic mobility of the protein was performed in cellogel strips as previously described ${ }^{12}$. Hemolysates treated with and without acidified serum were run in order to assess differences in mobility of the G6PD enzyme.

\section{Case report}

The patient, a 23 year old African American woman, presented with left upper abdominal pain, vomiting and blurry vision. She had a four year history of episodes of hemolysis, abdominal pain and dark urine. 
She was diagnosed with acute hemolytic exacerbation of PNH and was admitted. The patient was found to have anemia, leukocytosis and mild transaminitis. A Magnetic Resonance Venogram (MRV) of the abdomen and pelvis revealed several ill-defined low attenuation lesions of the posterior segment of the right hepatic lobe consistent with liver thrombosis so she was started on anticoagulation a concomitant urinary tract infection was treated with TrimethoprimSulfamethoxazole (Bactrim) and she was discharged.

After discharge, she followed up with a hematologist. On review of systems, the patient complained of a hemolytic attack with dark urine after taking Bactrim. On physical exam, she was found to have mildly icteric sclera. On further questioning of family history of other hematologic disorders, the patient said that one of her nephews has Glucose-6-Phosphate-Dehydrogenase deficiency. Because of her diagnosis of PNH and abdominal thrombosis, she was started on Eculizumab. The laboratory workup revealed a white blood cell count $4.6 \mathrm{k} / \mathrm{uL}, \mathrm{RBC}$ count of $3.8 \mathrm{k} / \mathrm{uL}$, hemoglobin of $11.8 \mathrm{~g} / \mathrm{dL}$, hematocrit $35 \%$, MCV $91 \mathrm{fl}$, platelets $327 \mathrm{k} / \mathrm{uL}$, reticulocytes $24.5 \%$, PTT 27.8, INR 1.7, D dimer $2.5 \mathrm{ug} / \mathrm{mL}$, bilirubin $1.3 \mathrm{mg} / \mathrm{dL}$, bilirubin direct $0.2 \mathrm{mg} / \mathrm{dL}$, bilirubin indirect $1.1 \mathrm{mg} / \mathrm{dL}$, ANC 2.7 k/uL, ALC 1.3 k/uL, G6PD screen normal, LDH 720 U/L. The flow cytometry at this point revealed: $69 \%$ of the red blood cells were partially deficient for CD59 and 9.1\% of the red blood cells were completely deficient for CD59; $86.3 \%$ of the granulocytes were partially deficient for CD59 and $6.4 \%$ were completely deficient for CD59.

\section{Results}

The family history and the case history suggested that the patient may have both G6PD deficiency and PNH, since the G6PD deficiency might explain the hemolysis precipitated by Bactrim, a drug that is reported to cause hemolysis in G6PD patients. Sequencing of DNA from her granulocytes confirmed that she was heterozygous for G6PD A- having a $G 6 P D B$ allele on one X-chromosome and a $G 6 P D A$ - allele on the other. This finding raised the question as to whether the somatic PIGA mutation causing her PNH took place on the X-chromosome carrying the $B$ or the $A$ - G6PD gene. The flow cytometry data showed that the patient most likely had $2 \mathrm{PNH}$ clones, a class I clone (partial deficiency) of about $86 \%$ and a class II clone (complete deficiency) of about $6 \%$. The HUMARA assay, which measures $\mathrm{X}$-inactivation, showed a single clone of about $90 \%$ (Figure 1), suggesting that in both clones the mutation had taken place on the same X-chromosome. We hypothesized that the mutations in PIGA would have taken place on the chromosome carrying the $G 6 P D A$ - allele since this would help explain the patient's reaction to Bactrim. Another factor was that treatment with eculizimab, by inhibiting lysis of PNH red cells may have led to a higher level of PNH (and concomitantly G6PD deficient) red cells than would be present in untreated patients. To determine which $G 6 P D$ allele was expressed in the PNH clone we sequenced cDNA from the patient's granulocytes. The sequencing trace showed that the vast majority of expressed $G 6 P D$ cDNA contained the wild type $(G 6 P D B)$ sequence at both nucleotides where it differs from G6PD A(Figure 2), leaving us to conclude that the PIGA mutations had taken place on the $\mathrm{X}$-chromosome containing the G6PD $B$ allele. This was confirmed at the protein level since the red blood cells lysed by acidified serum (the PNH cells) contained most of the G6PD activity while the residual cells did not contain detectable G6PD activity. While developing our hypothesis, which turned out to be incorrect, we also considered whether PNH/G6PD A- cells might have high levels of oxidative stress since both G6PD deficiency and PNH have been shown to be associated with elevated levels of reactive oxygen species ${ }^{11,13}$. We found that the patient's red blood cells contained ROS levels that were significantly higher than those from healthy controls, though surprisingly we did not detect any difference in ROS between PNH (CD55-) and normal (CD55+) cells (Figure 3).

\section{Digested}

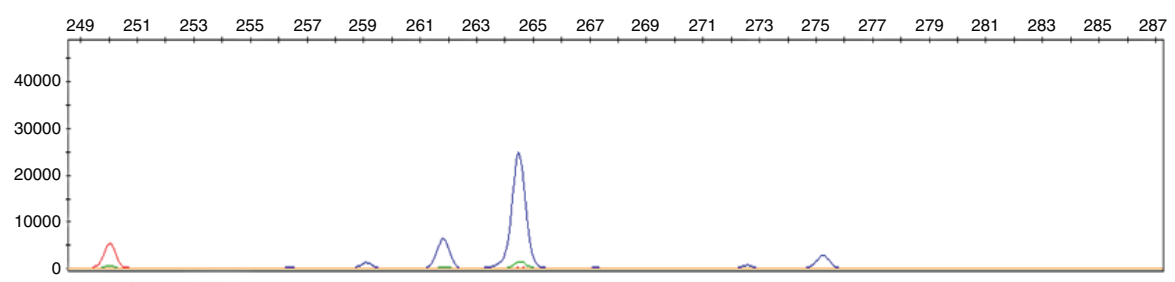

\section{Non-Digested}

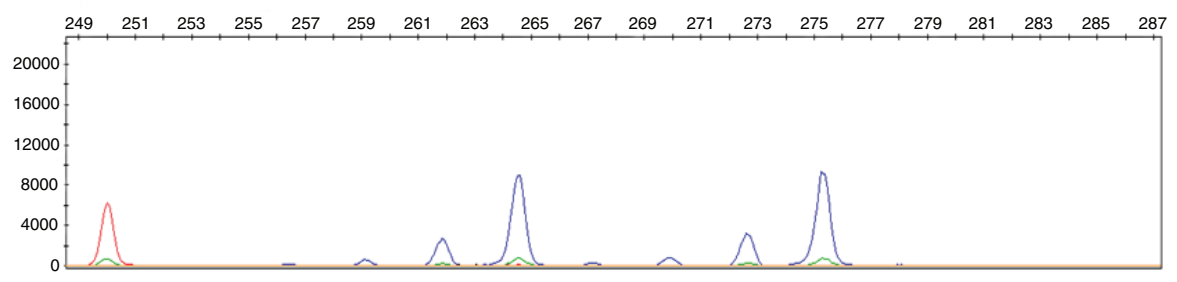

Figure 1. Clonal hematopoiesis in patient CHOP 277.01. The panel at the bottom shows the migration of the 2 microsatellite alleles in patient CHOP277.01 revealed by PCR analysis of a region of the Androgen receptor gene on the X-chromosome. The amplified region contains both a polymorphic repeat and a site for the methylation sensitive restriction enzyme Hhal. This site is methylated on the inactive X-chromosome. The top panel shows the same sample digested with Hhal before the PCR so only the fragment on the inactive X-chromosome is amplified. An imbalance in the allelic ratio reflects an imbalance in $\mathrm{X}$ inactivation and therefore indicates clonality. 


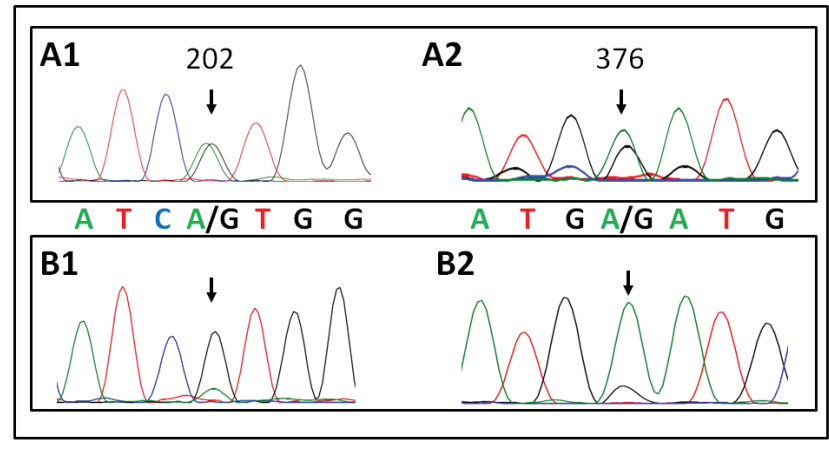

Figure 2. Patient CHOP 277.01 is heterozygous for G6PD B/Aand the $B$ allele is predominantly expressed. $A 1$ and $A 2$ shows sequencing of genomic DNA around the $202 \mathrm{G}->\mathrm{A}$ and the 376 $A->G$ mutation that lead to Val68Met and Asn126Asp changes in $\mathrm{G} 6 \mathrm{PD}$, respectively. Figures B1 and B2 show the CDNA sequence of the exact same mutations in the peripheral blood of the patient. The WT (B) allele is expressed in the majority of cells.

\section{Discussion}

$\mathrm{PNH}$ is a rare condition, having an incidence of about 1 in a million, so the co-incidental finding of a female with $\mathrm{PNH}$ and heterozygous for G6PD deficiency was an opportunity to observe the interaction between these 2 conditions which both involve red blood cell hemolysis mediated by X-linked genes. Notably the first demonstration that $\mathrm{PNH}$ was a clonal disease took advantage of a female $\mathrm{PNH}$ patient who was heterozygous for the electrophoretic variant $G 6 P D A^{14}$. In this patient both isozymes were present in a lysate of total red blood cells, but only one was present after acidified serum lysis, demonstrating clonality of the PNH cells.

In the case discussed here a female African American patient with PNH suffered episodes of hemolysis, often following treatment with Trimethoprim-Sulfamethoxazole (Bactrim), one of the drugs that is known to cause hemolysis in G6PD patients ${ }^{15}$. When it emerged that she was heterozygous for G6PD A-we hypothesized that her expanded PNH clones may be expressing only the G6PD A- protein. The hypothesis proved incorrect and the clone expressed the wild type G6PD allele. A possible explanation is that complement activation on G6PD A- red cells exposed to Bactrim might have triggered complement activation inducing the lysis of G6PD B PNH Type II red blood cells ${ }^{16}$. Alternatively since PNH patients often have several clones ${ }^{17}$ which change in prevalence it is possible that earlier she did have a G6PD A- clone but this was replaced by wild type clones. Finally we can also speculate that the combination of G6PD and PIGA deficiency confers a serious growth disadvantage and $\mathrm{PNH}$ clones in this situation are more likely to be G6PD wild type. There is no clear mechanism for this

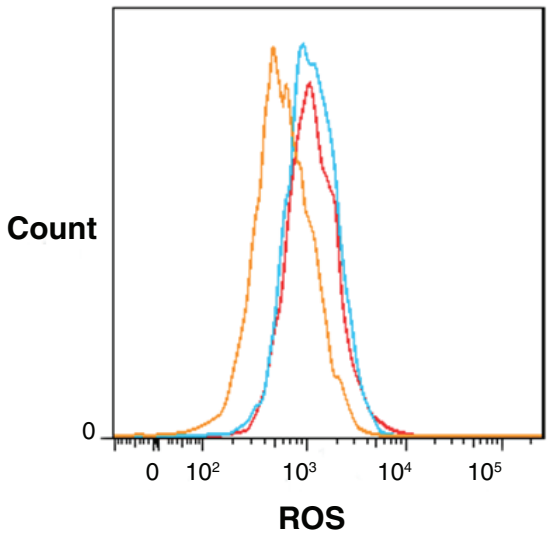

Figure 3. Flow cytometry analysis of the oxidative status of RBCs in the patient and a healthy donor. Peripheral blood mononuclear cells from the patient and a normal control were treated with the oxidation sensitive dye, CM-H2DCFDA, and the conversion to its oxidized fluorescent derivative assessed by flow cytometry. The fluorescence distribution histogram and the mean fluorescence channels (MFC) of each population derived from the normal control (orange) and the patient (blue for CD55 negative and red for CD55 positive cells) are shown.

however as $G 6 P D A$ - nucleated cells have similar enzyme activity to WT cells ${ }^{18}$ - the deficiency becoming apparent in red blood cells, which do not synthesize new protein ${ }^{19}$.

\section{Patient consent}

Written informed consent for publication of their clinical details was obtained from the patient.

\section{Author contributions}

PJM and MB conceived the study, NP designed the experiments and carried out the research. MM collected and collated clinical data. All authors contributed to preparing a draft of the manuscript and have agreed to the final content.

\section{Competing interests}

No competing interests were disclosed.

\section{Grant information}

The work has been supported by the Buck Family Endowed Chair in Hematology, and by NCI NIH grants 2R01CA106995 to PJ Mason, and 2R01 CA105312 to M Bessler.

The funders had no role in study design, data collection and analysis, decision to publish, or preparation of the manuscript.
1. Parker CJ: Paroxysmal nocturnal hemoglobinuria. Curr Opin Hematol. 2012; 19(3): 141-8.

PubMed Abstract | Publisher Full Text

2. Luzzatto L, Bessler M, Rotoli B: Somatic mutations in paroxysmal nocturnal hemoglobinuria: a blessing in disguise? Cell. 1997; 88(1): 1-4 PubMed Abstract | Publisher Full Text

3. McKeage K: Eculizumab: a review of its use in paroxysmal nocturnal haemoglobinuria. Drugs. 2011; 71(17): 2327-45

PubMed Abstract | Publisher Full Text

4. Kelly RJ, Hill A, Arnold LM, et al.: Long-term treatment with eculizumab in paroxysmal nocturnal hemoglobinuria: sustained efficacy and improved survival. Blood. 2011;117(25): 6786-92. survival. Blood. 2011; 117(25): 6786-92.
PubMed Abstract | Publisher Full Text

5. Mason PJ, Bautista JM, Gilsanz F: G6PD deficiency: the genotype-phenotype 
association. Blood Rev. 2007; 21(5): 267-83

PubMed Abstract | Publisher Full Text

6. Beutler E: G6PD deficiency. Blood. 1994; 84(11): 3613-36.

PubMed Abstract

7. Beutler E: G6PD: population genetics and clinical manifestations. Blood Rev. 1996; 10(1): 45-52.

PubMed Abstract | Publisher Full Text

8. Hirono A, Beutler E: Molecular cloning and nucleotide sequence of cDNA for human glucose-6-phosphate dehydrogenase variant A(-). Proc Natl Acad Sci U S A. 1988; 85(11): 3951-4.

PubMed Abstract | Publisher Full Text | Free Full Text

9. Au WY, Lam V, Pang A, et al.: Glucose-6-phosphate dehydrogenase deficiency in female octogenarians, nanogenarians, and centenarians. J Gerontol A Biol Sci Med Sci. 2006; 61(10): 1086-9.

PubMed Abstract | Publisher Full Text

10. Allen RC, Zoghbi HY, Moseley AB, et al:: Methylation of Hpall and Hhal sites nea the polymorphic CAG repeat in the human androgen-receptor gene correlates with X chromosome inactivation. Am J Hum Genet. 1992; 51(6): 1229-39. PubMed Abstract | Free Full Text

11. Amer J, Zelig O, Fibach E: Oxidative status of red blood cells, neutrophils, and platelets in paroxysmal nocturnal hemoglobinuria. Exp Hematol. 2008; 36(4): 369-77.

PubMed Abstract | Publisher Full Text

12. Rattazzi MC, Corash LM, van Zanen GE, et al:: G6PD deficiency and chronic hemolysis: four new mutants--relationships between clinical syndrome and enzyme kinetics. Blood. 1971; 38(2): 205-18.

PubMed Abstract
13. Ronquist G, Theodorsson E: Inherited, non-spherocytic haemolysis due to deficiency of glucose-6-phosphate dehydrogenase. Scand J Clin Lab Invest. 2007; 67(1): 105-11.

PubMed Abstract | Publisher Full Text

14. Oni SB, Osunkoya BO, Luzzatto L: Paroxysmal nocturnal hemoglobinuria: evidence for monoclonal origin of abnormal red cells. Blood. 1970; 36(2) 145-52.

PubMed Abstract

15. Serpa JA, Villarreal-Williams E, Giordano TP: Prevalence of G6PD deficiency in a large cohort of HIV-infected patients. J Infect. 2010; 61(5): 399-402. PubMed Abstract | Publisher Full Text

16. Arese P, Gallo V, Pantaleo A, et al.: Life and Death of Glucose-6-Phosphate Dehydrogenase (G6PD) Deficient Erythrocytes - Role of Redox Stress and Band 3 Modifications. Transfus Med Hemother. 2012; 39(5): 328-334. PubMed Abstract | Publisher Full Text | Free Full Text

17. Mortazavi Y, Merk B, Mclntosh J, et al.: The spectrum of PIG-A gene mutations in aplastic anemia/paroxysmal nocturnal hemoglobinuria (AA/PNH): a high incidence of multiple mutations and evidence of a mutational hot spot. Blood. 2003; 101(7): 2833-41.

PubMed Abstract | Publisher Full Text

18. Marks PA, Gross RT: Erythrocyte glucose-6-phosphate dehydrogenase deficiency: evidence of differences between Negroes and Caucasians with respect to this genetically determined trait. J Clin Invest. 1959; 38(12): 2253-62. PubMed Abstract | Publisher Full Text | Free Full Text

19. Mason PJ: New insights into G6PD deficiency. Br J Haematol. 1996; 94(4): 585-91.

PubMed Abstract 


\section{Open Peer Review}

\section{Current Peer Review Status: ? ?}

\section{Version 1}

Reviewer Report 04 September 2014

https://doi.org/10.5256/f1000research.5317.r6022

(c) 2014 Karadimitris A. This is an open access peer review report distributed under the terms of the Creative Commons Attribution License, which permits unrestricted use, distribution, and reproduction in any medium, provided the original work is properly cited.

\section{Anastasios Karadimitris}

Department of Haematology, Imperial College Healthcare NHS Trust and Imperial College, London, UK

Perdigones and colleagues describe the rare co-occurence in the same patient of two X-linked disorders, one acquired the other inherited, both causing intravascular haemolysis.

However how the interaction of the two disorders led to the clinical episode described in the case report is not clear because the temporal analysis of the clinical events and the associated laboratory tests are not presented in sufficient detail.

Charting clinical events, labs and therapeutic interventions might make association of the heamolytic episodes with co-trimoxazole or intercurrent infection clearer.

It appears that the majority of the PNH clone (in both granulocytes and red cells) is type II, i.e., only partially deficient of GPI. One assumes that this picture was obtained after the haemolytic attack. If so, it could be that type III, i.e., severely deficient RBC were indeed G6PD-deficient. Was flow-cytometry performed after Eculizumab treatment? Eculizumab would protect type III RBC from lysis and thus would allow re-assessment of G6PD activity.

\section{Minor points}

Was anti-CD55 or -CD59 was used for flow analysis? The authors state anti-CD55 in methods but describe anti-CD59 in results.

Normal ranges of lab tests need to be provided

'Another factor was that treatment with eculizumab, by inhibiting lysis of PNH red cells may have led to a higher level of PNH (and concomitantly G6PD deficient) red cells than would be present in untreated patients.' This statement is rather irrelevant because eculizumab was started after treatment with co-trimoxazole.

Need to use Greek characters appropriately, i.e., ul should be ml 
Competing Interests: No competing interests were disclosed.

I confirm that I have read this submission and believe that I have an appropriate level of expertise to confirm that it is of an acceptable scientific standard, however I have significant reservations, as outlined above.

Author Response ( F1000Research Advisory Board Member ) 15 Oct 2014

Philip Mason, Abramson Pediatric Research Center, The Children's Hospital of Philadelphia, USA

It appears that the majority of the PNH clone (in both granulocytes and red cells) is type II, i.e., only partially deficient of GPI. One assumes that this picture was obtained after the haemolytic attack. If so, it could be that type III, i.e., severely deficient RBC were indeed G6PD-deficient. Was flow-cytometry performed after Eculizumab treatment? Eculizumab would protect type III RBC from lysis and thus would allow re-assessment of G6PD activity.

We agree with this comment. Of course it is possible that at the time of the observation of association of co-trimoxazole associated hemolysis a different PNH clone was more prevalent than when analyzed for G6PD deficiency. We included this possibility in our discussion. The most recent flow cytometry was performed when the patient was on eculizimab.

\section{Minor points}

Was anti-CD55 or -CD59 was used for flow analysis? The authors state anti-CD55 in methods but describe anti-CD59 in results. The diagnosis for PNH was performed in a CLIA approved clinical laboratory of the hospital.

We present the results for CD59. CD55 was also tested however the discrimination between the three populations is more difficult.

Normal ranges of lab tests need to be provided.

We have included normal ranges for laboratory tests in parentheses after the patient's values.

Another factor was that treatment with eculizumab, by inhibiting lysis of PNH red cells may have led to a higher level of PNH (and concomitantly G6PD deficient) red cells than would be present in untreated patients.' This statement is rather irrelevant because eculizumab was started after treatment with co-trimoxazole.

We think this statement is valid because treatment with eculizimab increases the level of PIGA deficient red cells. On our hypothesis that the G6PDA- allele was linked with PIGA- then PIGA-,G6PDA- red cells would increase. Our hypothesis however was not correct.

Need to use Greek characters appropriately, i.e., ul should be ml 
We have corrected our incorrect use of Greek characters.

Competing Interests: No competing interests were disclosed.

Reviewer Report 28 August 2014

https://doi.org/10.5256/f1000research.5317.r5816

(C) 2014 Notaro R. This is an open access peer review report distributed under the terms of the Creative Commons Attribution License, which permits unrestricted use, distribution, and reproduction in any medium, provided the original work is properly cited.

\section{Rosario Notaro}

Laboratory of Genetics and Gene Transfer, Core Research Laboratory, Istituto Toscano Tumori, Florence, Italy

Perdigones and collaborators report an interesting clinical case about the association of PNH and G6PD deficiency. However, the timing of the clinical history is not clear and the report is scanty of some relevant clinical/laboratory data.

\section{Major scientific points.}

1. The Authors should provide the exact timing of entire clinical history: diagnosis; infective episode resulting in the prescription of trimethoprim-sulfamethoxazole -co-trimoxazole(which dose?); start and stop of co-trimoxazole; "dark urine episode" associated with cotrimoxazole; start of eculizumab; time of biological studies.

2. It is extremely important that the Authors provide more details about the timing and the features of the hemolytic attack apparently associated with co-trimoxazole: how long after the start of co-trimoxazole treatment the patient experienced the "dark urine episode"? There are any objective data at the time of this "dark urine episode" or the episode has been just self-reported? At the time of this "dark urine episode" there were any signs/symptoms of an ongoing infective condition?

3. The Authors provide clinical/laboratory details only at one time point, that seems be after the start of eculizumab (how long after?). They should provide such clinical/laboratory details at time of diagnosis, at the time of the co-trimoxazole associated "hemolytic attack", at start of eculizumab treatment, etc.: blood count, absolute reticulocyte count, LDH levels (providing the normal range), flow citometry, etc.

4. In PNH patients the presence of red blood cells with partial deficiency of GPI-linked molecules is relatively common. However, the presence of granulocyte/monocyte with partial deficiency of GPI-linked molecules is uncommon: thus, it would be interesting to show the dot plot of the "CD59/lineage marker" analysis of granulocytes and monocytes of 
this patient.

5. The Authors, at variance with their starting hypothesis, have clearly proven that in this patient PNH cells express the wild type G6PD B. They provide 2 possible explanations for the co-trimoxazole-associated "hemolytic crisis" observed in this patient. These hypotheses are interesting but their probability is very low. I suggest that the Authors should discuss a much more likely explanation: this "hemolytic crisis" was just due to the infective condition that led to the prescription of co-trimoxazole.

\section{Minor points}

1. In the Introduction the Authors report the classical classification of PNH cells as Type III (completely deficient in GPI anchored proteins), Type II (partially deficient) and Type I (normal display of GPI-linked proteins). However, in the Results they write about "class I clone (partial deficiency) ... and class II clone (complete deficiency) ": this is extremely confusing.

2. In the Results the 2 sentences (from "We hypothesized that ..." to "...red cells than would be present in untreated patients.") seem to suggest that the relative expansion of the "PNH (and concomitantly G6PD deficient) red cells" following eculizumab treatment could have played a role in the co-trimoxazole associated hemolytic attack. The Authors should rephrase these sentences since eculizumab has been started after the co-trimoxazole associated hemolytic attack.

3. Trimethoprim-sulfamethoxazole (co-trimoxazole) is a generic drug name thus it should not be capitalized. In the Results, "eculizumab" should not be capitalized.

4. The brand name Bactrim should be replaced with the generic drug name.

5. The levels of ROS in the patient red cells should be compared with a group of healthy controls: the comparison with only one control does not allow any conclusion.

6. The Authors should report the concentration of DCF used for ROS detection.

Competing Interests: No competing interests were disclosed.

\section{I confirm that I have read this submission and believe that I have an appropriate level of expertise to confirm that it is of an acceptable scientific standard, however I have significant reservations, as outlined above.}

Author Response ( F1000Research Advisory Board Member) 15 Oct 2014

Philip Mason, Abramson Pediatric Research Center, The Children's Hospital of Philadelphia, USA

Perdigones and collaborators report an interesting clinical case about the association of PNH and G6PD deficiency. However, the timing of the clinical history is not clear and the report is scanty of 
some relevant clinical/laboratory data.

\section{Major scientific points.}

1. The Authors should provide the exact timing of entire clinical history: diagnosis; infective episode resulting in the prescription of trimethoprim-sulfamethoxazole -co-trimoxazole(which dose?); start and stop of co-trimoxazole; "dark urine episode" associated with cotrimoxazole; start of eculizumab; time of biological studies.

In our revised manuscript we include a fuller, more detailed case history. Of note is the fact that the patient presented to the specialty clinic with a probably 6 year history of PNH and that most of the patients past history relies on records and notes. Here we demonstrate that the patient has indeed both conditions PNH and G6PD deficiency. With these results in hand we try to explain the patient's history and clinical observations. It was not our intention to prove that indeed the hemolysis described by the emergency physician after co-trimoxazole was indeed triggered by the drug, but rather to evaluate and discuss the possibility as the patient carries on her chart the diagnosis of co-trimoxazole hypersensitity due to this incident. We try to make this clearer in the case description. We hope this will answer the criticism of the reviewer.

2. It is extremely important that the Authors provide more details about the timing and the features of the hemolytic attack apparently associated with co-trimoxazole: how long after the start of co-trimoxazoletreatment the patient experienced the "dark urine episode"? There are any objective data at the time of this "dark urine episode" or the episode has been just self-reported? At the time of this "dark urine episode" there were any signs/symptoms of an ongoing infective condition?

The discussion above and the revised version provides many more details.

3. The Authors provide clinical/laboratory details only at one time point, that seems be after the start of eculizumab (how long after?). They should provide such clinical/laboratory details at time of diagnosis, at the time of the co-trimoxazole associated "hemolytic attack", at start of eculizumab treatment, etc.: blood count, absolute reticulocyte count, LDH levels (providing the normal range), flow citometry, etc.

We have provided all the history we can obtain in the revised version.

4. In PNH patients the presence of red blood cells with partial deficiency of GPI-linked molecules is relatively common. However, the presence of granulocyte/monocyte with partial deficiency of GPI-linked molecules is uncommon: thus, it would be interesting to show the dot plot of the "CD59/lineage marker" analysis of granulocytes and monocytes of this patient.

The diagnosis of PNH and the subtype analysis of PNH granulocytes was performed in a CLIA approved clinical laboratory, and their test results are reported here. The 
individual dot blots are not available to us. However the senior author who was involved in the setup and quality assessment of flow-cytometric PNH testing at this institution fully trusts their analysis. We agree with the reviewer that partial GPIanchor deficiency in granulocytes is less frequently observed and very much depends on the underlying mutation and the antibody chosen for the analysis.

5. The Authors, at variance with their starting hypothesis, have clearly proven that in this patient PNH cells express the wild type G6PD B. They provide 2 possible explanations for the co-trimoxazole-associated "hemolytic crisis" observed in this patient. These hypotheses are interesting but their probability is very low. I suggest that the Authors should discuss a much more likely explanation: this "hemolytic crisis" was just due to the infective condition that led to the prescription of co-trimoxazole.

We agree that this is a possible alternative explanation and have added this in the discussion however considering the rather bland urine sediment we actually favor the other two possible explanations.

\section{Minor points}

1. In the Introduction the Authors report the classical classification of PNH cells as Type III (completely deficient in GPI anchored proteins), Type II (partially deficient) and Type I (normal display of GPI-linked proteins). However, in the Results they write about "class I clone (partial deficiency) ... and class II clone (complete deficiency) ": this is extremely confusing.

This has been corrected

2. In the Results the 2 sentences (from "We hypothesized that ..." to "...red cells than would be present in untreated patients.") seem to suggest that the relative expansion of the "PNH (and concomitantly G6PD deficient) red cells" following eculizumab treatment could have played a role in the co-trimoxazole associated hemolytic attack. The Authors should rephrase these sentences since eculizumab has been started after the cotrimoxazole associated hemolytic attack.

The timing of the eculizimab treatment and the hemolysis are given in the detailed case report that we now provide.

3. Trimethoprim-sulfamethoxazole (co-trimoxazole) is a generic drug name thus it should not be capitalized. In the Results, "eculizumab" should not be capitalized.

We have corrected this.

4. The brand name Bactrim should be replaced with the generic drug name.

We have corrected this.

5. The levels of ROS in the patient red cells should be compared with a group of healthy 
controls: the comparison with only one control does not allow any conclusion.

The experiment was carried out with several controls and it confirms the results of Amer et al., cited as our ref 14 that ROS are elevated in $\mathrm{PNH}$. Our point is a small one, that we might expect a further elevation if we compare PNH and normal cells from this patient, if indeed PIGA- is linked with G6PDA-. We didn't find this. We include the sentence "The figure shows a representative example of 4 normal controls that gave similar results" to clarify this.

6. The Authors should report the concentration of DCF used for ROS detection.

We have done this.

Competing Interests: No competing interests were disclosed.

The benefits of publishing with F1000Research:

- Your article is published within days, with no editorial bias

- You can publish traditional articles, null/negative results, case reports, data notes and more

- The peer review process is transparent and collaborative

- Your article is indexed in PubMed after passing peer review

- Dedicated customer support at every stage

For pre-submission enquiries, contact research@f1000.com 